\title{
THE UNUSUALLY LONG DURATION GAMMA-RAY BURST GRB 000911: DISCOVERY OF THE AFTERGLOW AND HOST GALAXY ${ }^{1}$
}

\author{
P. A. Price, ${ }^{2,3}$ E. Berger, ${ }^{2}$ S. R. Kulkarni, ${ }^{2}$ S. G. Duorgovski ${ }^{2}$ D. W. Fox,${ }^{2}$ A. Mahabal,${ }^{2}$ K. Hurley,${ }^{4}$ J. S. Bloom, ${ }^{2}$ \\ D. A. Frail, ${ }^{5}$ T. J. Galama, ${ }^{2}$ F. A. Harrison, ${ }^{2}$ G. Morrison, ${ }^{6}$ D. E. Reichart, ${ }^{2}$ S. A. Yost, ${ }^{2}$ R. Sari, ${ }^{7}$ T. S. Axelrod ${ }^{3}$ \\ T. Cline, ${ }^{8}$ S. Golenetskit, ${ }^{9}$ E. Mazets, ${ }^{9}$ B. P. Schmidt, ${ }^{3}$ And J. TrombKa ${ }^{8}$ \\ Received 2001 October 12; accepted 2002 March 10
}

\begin{abstract}
Of all the well-localized gamma-ray bursts, GRB 000911 has the longest duration $\left(T_{90}=500 \mathrm{~s}\right)$ and ranks in the top 1\% of BATSE bursts for fluence. Here we report the discovery of the afterglow of this unique burst. In order to simultaneously fit our radio and optical observations, we are required to invoke a model involving a hard electron distribution, $p \sim 1.5$, and a jet-break time less than 1.5 days. A spectrum of the host galaxy taken 111 days after the burst reveals a single emission line, interpreted as [O II] at a redshift $z=1.0585$, and a continuum break that we interpret as the Balmer limit at this redshift. Despite the long $T_{90}$, the afterglow of GRB 000911 is not unusual in any other way when compared to the set of afterglows studied to date. We conclude that the duration of the GRB plays little part in determining the physics of the afterglow.

Subject headings: galaxies: high-redshift — gamma rays: bursts

On-line material: color figures
\end{abstract}

\section{INTRODUCTION}

The revolution in understanding the origin of gamma-ray bursts (GRBs) has been driven by the discovery and exploitation of long-lived lower energy "afterglow" phenomena (Costa et al. 1997; van Paradijs et al. 1997; Frail et al. 1997). However, this revolution has been restricted to GRBs belonging to the so-called long/soft subclass (Kouveliotou et al. 1993), which are GRBs with duration $T_{90}>2 \mathrm{~s}$; here $T_{90}$ is the duration of the 90th percentile of the fluence. Technical difficulties have prevented rapid and precise localization of short bursts, and consequently these objects continue to remain mysterious (see Hurley et al. 2002 for a summary of the searches for afterglows of short-duration GRBs).

The currently popular theory for GRBs holds that the long/soft GRBs come from the collapse of massive stars (MacFayden \& Woosley 1999) and short/hard GRBs are due to the merger of a pair of compact objects (e.g., Fryer et al. 1999). The former model naturally accounts for the long duration since the newly formed black hole has access to a large amount of stellar material, whereas in the latter model, the duration of the accretion process is limited by the small

${ }^{1}$ Partially based on observations obtained at the W. M. Keck Observatory, which is operated by the California Association for Research in Astronomy, a scientific partnership among the California Institute of Technology, the University of California, and the National Aeronautics and Space Administration.

2 Palomar Observatory, MS 105-24, California Institute of Technology, Pasadena, CA 91125

${ }^{3}$ Research School of Astronomy and Astrophysics, Mount Stromlo Observatory, Cotter Road, Weston, ACT 2611, Australia.

${ }^{4}$ Space Sciences Laboratory, University of California, Berkeley, CA 94720.

${ }^{5}$ National Radio Astronomy Observatory, P.O. Box O, Socorro, NM 87801.

${ }^{6}$ Infrared Processing and Analysis Center, MS 100-22, California Institute of Technology, Pasadena, CA 91125.

7 Theoretical Astrophysics, MS 130-33, California Institute of Technology, Pasadena, CA 91125.

8 NASA Goddard Space Flight Center, Greenbelt, MD 20771

${ }^{9}$ Ioffe Physico-Technical Institute, Saint Petersburg 194021, Russia. amount of matter left over following such a merger (e.g., Janka et al. 1999; Narayan, Piran, \& Kumar 2001).

Regardless of our current theoretical prejudice, the following three phenomena give us the most intimate observational view of the central engine: the variability, the duration of GRB profiles, and the opening angles of jets. In the "internal-external" shock model for long-duration GRBs, a model heavily supported by observations, the variability and the duration have been argued to arise from different processes (Nakar \& Piran 2002). The recent observational evidence for significant diversity in jet opening angles (Frail et al. 2001) has motivated preliminary theoretical forays into the same (van Putten 2001).

Unfortunately, we are far from knowing how to translate these three properties (variability, duration, and opening angles) into physical properties of the central engine. In contrast, the study of the afterglow yields good measures of bulk properties (explosion energy, ambient density, location within the host galaxy, the host galaxy itself) as well as potentially direct information about the progenitors (X-ray line features, late-time red bumps potentially related to an underlying supernova). Thus, the next natural step in GRB studies will be to start empirically relating the engine properties to the afterglow properties. It is in this context that the study of GRB 000911 with its unusually long duration, $T_{90}=500 \mathrm{~s}$ (the longest GRB with an identified afterglow to date), becomes potentially interesting. Here we present the gamma-ray time history, the discovery of the afterglow, broadband modeling of the afterglow, and observations of the host galaxy of GRB 000911.

\section{GAMMA-RAY OBSERVATIONS}

GRB 000911 was observed by three instruments in the Interplanetary Network (IPN): Ulysses GRB (25-150 keV), Konus/Wind (10-10,000 keV), and the Near-Earth Asteroid Rendezvous X- and Gamma-Ray Spectrometer (NEAR/ XGRS; $100-1000 \mathrm{keV})$. The Earth-crossing time of the 


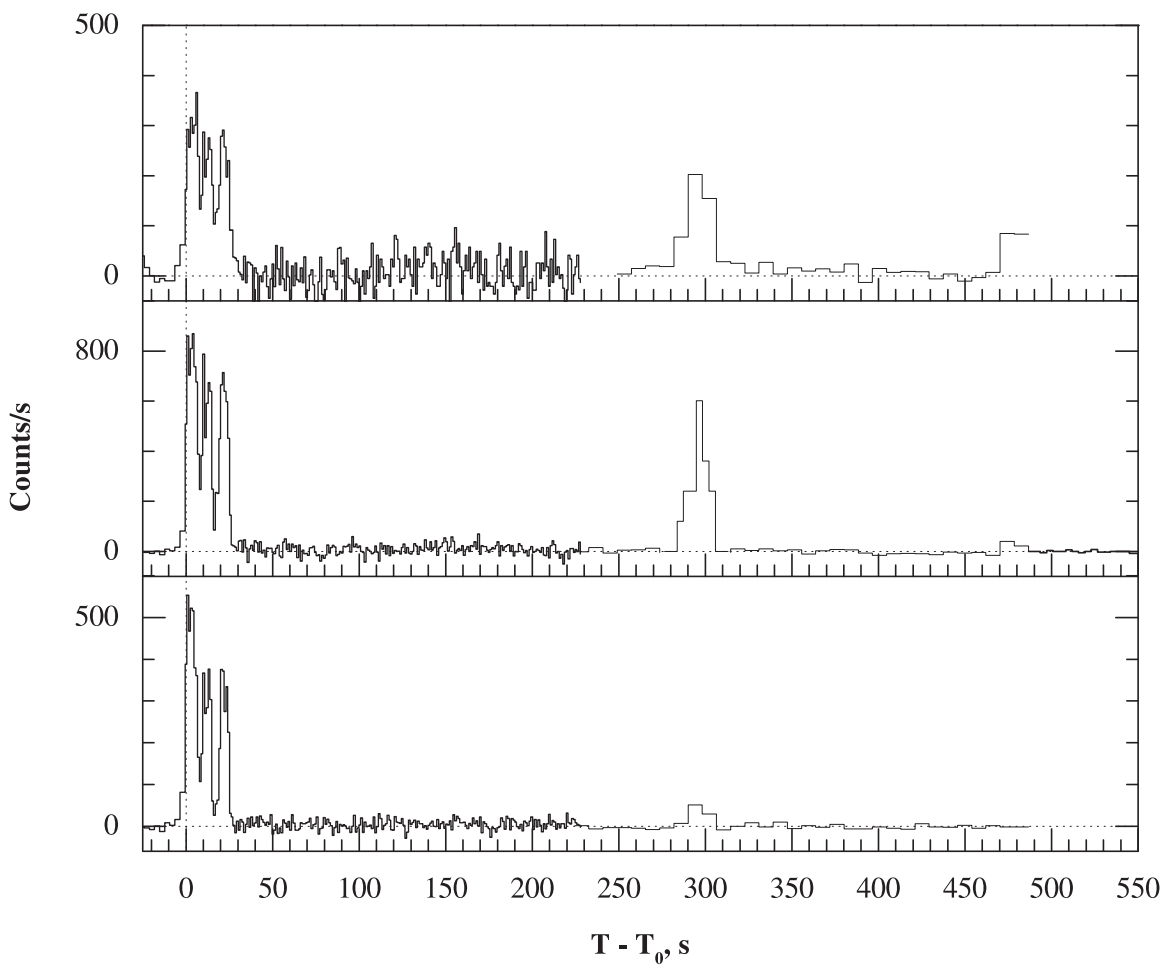

FIG. 1.-Gamma-ray light curves for GRB 000911 in three energy ranges, from the Konus/Wind experiment. From top to bottom, the energies are 18-71, 71-306, and 306-1150 keV, respectively.

burst was 2000 September 11.30242 UT. By 22.8 hours after the burst, the event was localized to an area of $30 \mathrm{arcmin}^{2}$ and reported promptly to the GRB Coordinates Network $(\mathrm{GCN})^{10}$ (Hurley et al. 2001).

\footnotetext{
${ }^{10}$ Available at http://gcn.gsfc.nasa.gov.
}

Three distinct episodes (pulses) can be seen in the Konus light curves (Fig. 1). All three episodes are also visible in the Ulysses data, but the third is only marginally significant in the NEAR time history, indicating that the energy spectrum is undergoing the familiar hard-to-soft evolution. The Konus energy spectra (Fig. 2) show this more clearly. The fact that the three pulses occur at the same relative times in
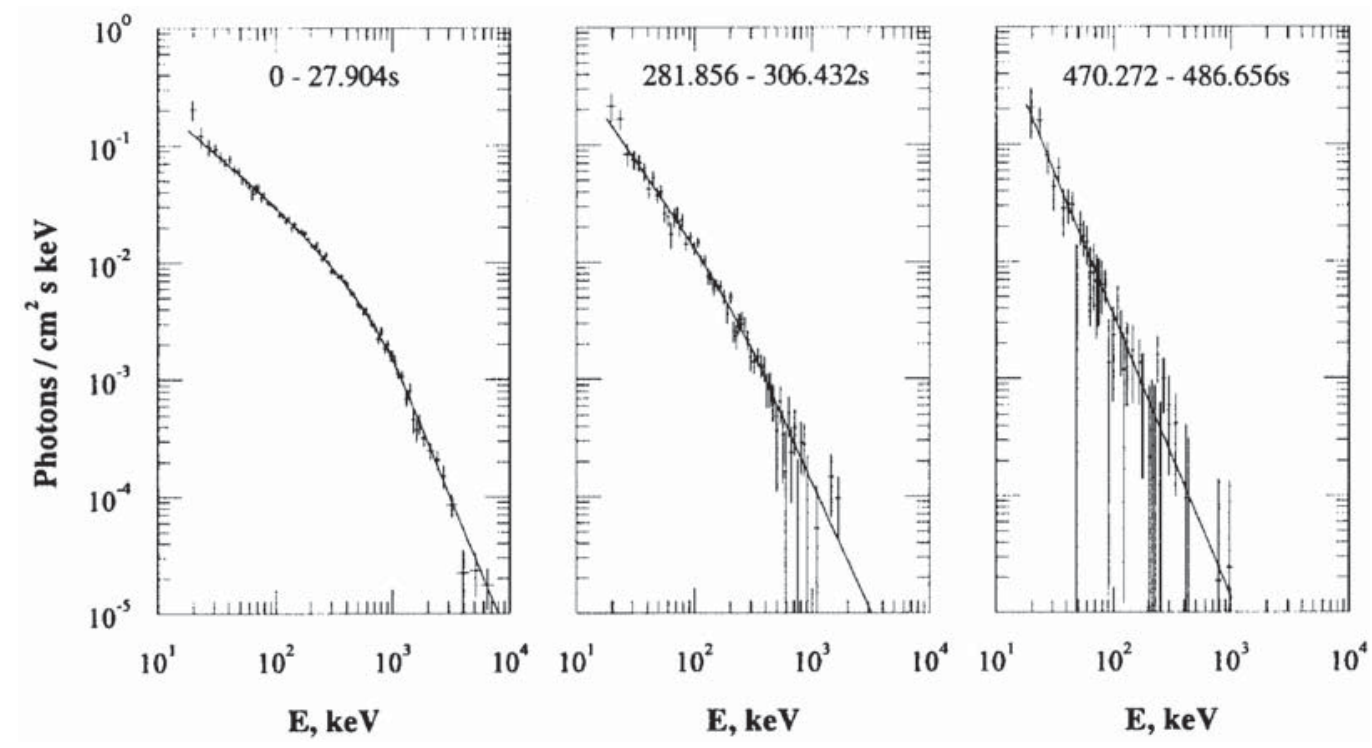

FIg. 2.-Konus / Wind spectra of the three pulses of GRB 000911. The three pulses are indicated in Fig. 1. The epochs over which the spectra were extracted are displayed inside the figure. The first two spectra were fitted to a Band et al. (1993) model. There were not enough counts in the third pulse, and consequently the fit was restricted to a simple power-law model. Fitted parameters are $\alpha=-0.84$ and -1.38 and $E_{0}=850$ and $450 \mathrm{keV}$ for the first and second pulses, and $\beta=-2.43,-2.22$, and -2.4 for pulses $1-3$, respectively. 


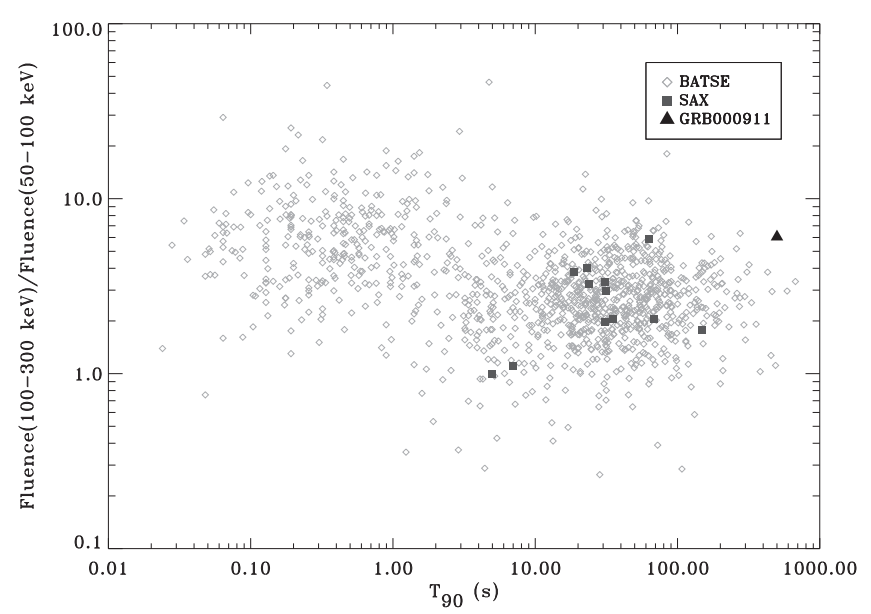

FIG. 3.- Hardness-duration plot for 1676 BATSE GRBs (diamonds; Paciesas et al. 2000), demonstrating the two classes of GRBs (Kouveliotou et al. 1993). Squares represent BATSE GRBs for which afterglows were identified with BeppoSAX. It is clear that each belongs to the long/soft class. The triangle ( far right) represents GRB 000911, which has the longest duration of all GRBs with observed afterglows. [See the electronic edition of the Journal for a color version of this figure.]

the Ulysses and Konus time histories proves that they are all from the same direction and makes it unlikely that the profiles are due to two distinct GRBs that are coincidentally detected at about the same time. Thus, we conclude that the duration of this burst is approximately $500 \mathrm{~s}$ as measured from the Ulysses $25-150 \mathrm{keV}$ data (with estimates ranging from 488 to $510 \mathrm{~s}$ based on the choice of background), making it the longest duration GRB whose afterglow has been studied to date (see Fig. 3) ${ }^{11}$ and placing it in the top $0.1 \%$ of the GRBs observed by BATSE.

The fluences of the three pulses are given in Table 1. With a total fluence of approximately $2.3 \times 10^{-4} \mathrm{ergs} \mathrm{cm}^{-2}$ and a $50-300 \mathrm{keV}$ fluence of approximately $4.3 \times 10^{-5} \mathrm{ergs} \mathrm{cm}^{-2}$, this burst is within the top $1 \%$ of the GRBs observed by BATSE.

\section{DISCOVERY OF THE OPTICAL AND RADIO AFTERGLOW}

The IPN error box was first observed by the robotic 50 inch $(1.3 \mathrm{~m})$ telescope at Mount Stromlo Observatory (MSO) with the MACHO dual-beam camera 1.4 days after the burst. Very Large Array (VLA) ${ }^{12}$ observations of GRB 000911 were initiated 0.8 days later at $8.46 \mathrm{GHz}$. All radio observations were obtained in the standard continuum mode with $2 \times 50 \mathrm{MHz}$ contiguous bands. We observed the extragalactic source $\mathrm{J} 0203+115$ for phase calibration and 3C $48(\mathrm{~J} 0137+331)$ for flux calibration. All data were reduced using the Astronomical Image Processing System (AIPS).

In the earliest radio observations (2.2 and 3.1 days after the burst), we identified as a candidate afterglow a source located at coordinates R.A. $=02^{\mathrm{h}} 18^{\mathrm{m}} 34^{\mathrm{s}} .36$, decl. $=$

\footnotetext{
11 Data for Fig. 3 from catalog at http://cossc.gsfc.nasa.gov/batse/ BATSE_Ctlg.

12 The National Radio Astronomy Observatory (NRAO) is a facility of the National Science Foundation operated under cooperative agreement by Associated Universities, Inc. NRAO operates the VLA.
}

TABLE 1

Fluences of the Three Pulses

\begin{tabular}{cccc}
\hline \hline $\begin{array}{c}\text { Epoch } \\
(\mathrm{s})\end{array}$ & $\begin{array}{c}\text { Fluence } \\
\left(\mathrm{ergs} \mathrm{cm}^{-2}\right)\end{array}$ & $\begin{array}{c}\text { Peak Flux } \\
\left(\mathrm{ergs} \mathrm{cm}^{-2} \mathrm{~s}^{-1}\right)\end{array}$ & $\begin{array}{c}\text { Energy Interval } \\
(\mathrm{keV})\end{array}$ \\
\hline $0-27.9 \ldots \ldots \ldots \ldots$. & $2.0 \times 10^{-4}$ & $2 \times 10^{-5}$ & $15-8000$ \\
$281.9-306.4 \ldots \ldots$. & $2.7 \times 10^{-5}$ & $\ldots$ & $15-5000$ \\
$470.3-486.7 \ldots \ldots$. & $4.0 \times 10^{-6}$ & $\ldots$ & $15-1000$ \\
\hline
\end{tabular}

Note.-Peak fluxes for the second and third pulses are not shown because the time bins were too large: $8.192 \mathrm{~s}$.

$+07^{\circ} 44^{\prime} 27^{\prime \prime} 65$ (J2000.0) (with an estimated $2 \sigma$ error of 0 ". 6 in each axis) that brightened by $2 \sigma$. An optical counterpart to this radio source (Fig. 4) with $R \sim 20.6$ mag was identified in our images obtained from the 50 inch MSO telescope, but the source was not present on the Digital Palomar Observatory Sky Survey (Djorgovski et al. 1999), although this is near the plate limit. Subsequent observations with the Echellette Spectrograph and Imager (ESI) on the Keck II telescope of the W. M. Keck Observatory showed that the source decayed and thereby confirmed the identification of this source as the afterglow of GRB 000911.

Subsequently, we began a program of VLA observations of the radio afterglow. The log of radio observations and the fluxes of the radio afterglow can be found in Table 2 .

We observed the optical afterglow at the Keck Observatory in $R$ and $I$ bands at three additional epochs after the discovery. We bias-subtracted and flat-fielded the optical observations in the usual way, using the Image Reduction

TABLE 2

RADIO OBSERVATIONS OF GRB 000911 MADE With the Very Large Array

\begin{tabular}{|c|c|c|}
\hline $\begin{array}{c}\text { Epoch } \\
\text { (UT) }\end{array}$ & $\begin{array}{c}\nu_{0} \\
(\mathrm{GHz})\end{array}$ & $\begin{array}{l}S \pm \sigma \\
(\mu \mathrm{Jy})\end{array}$ \\
\hline 2000 Sep $13.49 \ldots \ldots$. & 8.46 & $165 \pm 60$ \\
\hline 2000 Sep $14.36 \ldots \ldots$ & 8.46 & $278 \pm 36$ \\
\hline 2000 Sep $15.36 \ldots \ldots$ & 4.86 & $60 \pm 34$ \\
\hline 2000 Sep $15.36 \ldots \ldots$ & 8.46 & $230 \pm 34$ \\
\hline 2000 Sep $17.49 \ldots \ldots$ & 4.86 & $65 \pm 25$ \\
\hline 2000 Sep $17.49 \ldots \ldots$. & 8.46 & $90 \pm 22$ \\
\hline 2000 Sep $22.35 \ldots \ldots$. & 4.86 & $71 \pm 23$ \\
\hline 2000 Sep $22.35 \ldots \ldots$. & 8.46 & $88 \pm 25$ \\
\hline 2000 Sep $27.16 \ldots \ldots$. & 8.46 & $110 \pm 43$ \\
\hline 2000 Sep $28.50 \ldots \ldots$. & 4.86 & $27 \pm 31$ \\
\hline 2000 Sep $28.50 \ldots \ldots$. & 8.46 & $42 \pm 23$ \\
\hline 2000 Oct $2.22 \ldots \ldots .$. & 4.86 & $10 \pm 25$ \\
\hline 2000 Oct $2.22 \ldots \ldots .$. & 8.46 & $80 \pm 26$ \\
\hline 2000 Oct $4.23 \ldots \ldots .$. & 8.46 & $54 \pm 24$ \\
\hline 2000 Oct $7.29 \ldots \ldots$. & 8.46 & $-21 \pm 66$ \\
\hline 2000 Oct $8.29 \ldots \ldots$. & 8.46 & $-6 \pm 50$ \\
\hline 2000 Oct $10.27 \ldots \ldots$ & 8.46 & $-46 \pm 81$ \\
\hline 2000 Oct $14.24 \ldots \ldots$. & 8.46 & $45 \pm 26$ \\
\hline 2000 Nov $7.38 \ldots \ldots .$. & 8.46 & $30 \pm 30$ \\
\hline 2001 Jan $15.08 \ldots \ldots .$. & 8.46 & $26 \pm 18$ \\
\hline
\end{tabular}

Note.-The columns are (left to right), UT date of the start of each observation, observing frequency, and peak flux density at the best-fit position of the radio transient, with the error given as the rms noise on the image. 

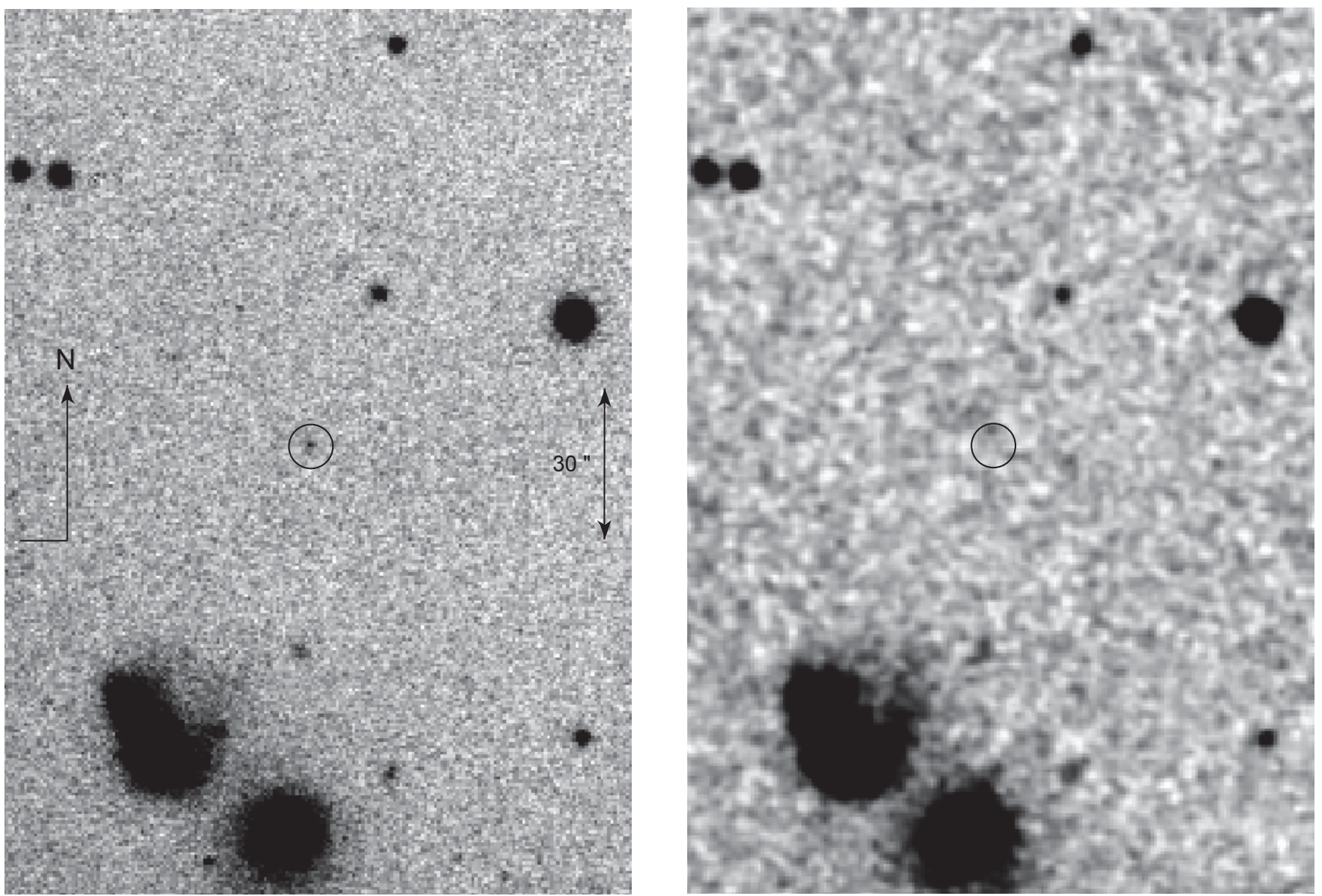

FIG. 4.- Identification of the optical afterglow of GRB 000911. Left: Image of $R_{M}$ from the 50 inch telescope at MSO. Right: Corresponding image from the DPOSS $F$ plate. The location of the optical afterglow is circled.

and Analysis Facility (IRAF). ${ }^{13}$ Aperture photometry was used to measure the magnitude of the optical afterglow in each image. Photometric zero points for the Keck imaging were based on stars in the field, with calibrated values provided by S. Covino and D. Lazzati from their first Very Large Telescope (VLT) epoch (Lazzati et al. 2001). In addition, the large range of the color of stars in the field $[(R-I) \sim 0.4-1.8 \mathrm{mag}]$ allowed us to fit and apply color terms in order to correct bandpass mismatches.

The first-epoch optical images (in MACHO blue and red bands) were not sufficiently deep to use the calibrated VLT stars. For these images, we transformed the stellar magnitudes calibrated by Henden (2000) to the MACHO photometric system using (the inverse of) the transformation calculated by Bessell \& Germany (1999). We then photometered the afterglow against these stars and transformed back to the Johnson-Cousins system. An additional error of 3\% was added in quadrature to these measurements to reflect errors in the transformation.

The log of our optical observations and the photometry are displayed in Table 3. All the optical data, photometric and spectroscopic, when displayed in physical units (Jy), have been corrected for foreground Galactic reddening

13 IRAF is distributed by the National Optical Astronomy Observatory.
$E_{B-V}=0.107$ mag (Schlegel, Finkbeiner, \& Davis 1998) and an average Galactic extinction curve (with $R_{V}=3.1$ ) modeled by Cardelli, Clayton, \& Mathis (1989).

Optical and radio light curves are displayed in Figures 5 and 6, respectively. The optical light curves (which include data from Lazzati et al. 2001) exhibit power-law decay, $f(t) \propto t^{-\alpha}$ with $\alpha=1.46 \pm 0.05$, followed by a leveling (at about 15 days). We identified the late-time steady source with the host galaxy of GRB 000911.

TABLE 3

Optical Observations of the Afterglow of GRB 000911

\begin{tabular}{|c|c|c|c|}
\hline $\begin{array}{l}\text { Date } \\
\text { (UT) }\end{array}$ & Filter & Mag & Telescope \\
\hline 2000 Sep $12.737 \ldots \ldots$. & $V$ & $20.90 \pm 0.10$ & MSO 50 inch \\
\hline 2000 Sep $12.737 \ldots \ldots$. & $R$ & $20.700 \pm 0.081$ & MSO 50 inch \\
\hline 2000 Sep $15.593 \ldots \ldots .$. & $R$ & $22.299 \pm 0.030$ & Keck II + ESI \\
\hline 2000 Sep $15.606 \ldots \ldots$. & $I$ & $21.816 \pm 0.052$ & Keck II + ESI \\
\hline 2000 Sep $25.421 \ldots \ldots .$. & $R$ & $24.052 \pm 0.094$ & Keck I + LRIS \\
\hline 2000 Sep $25.451 \ldots \ldots .$. & $I$ & $23.412 \pm 0.071$ & Keck I + LRIS \\
\hline 2001 Jan $1.221 \ldots \ldots \ldots$ & $R$ & $25.414 \pm 0.119$ & Keck II + ESI \\
\hline 2001 Jan $1.248 \ldots \ldots \ldots$ & $I$ & $24.415 \pm 0.083$ & Keck II + ESI \\
\hline
\end{tabular}

NoTE.- These measurements have not been corrected for Galactic extinction. 


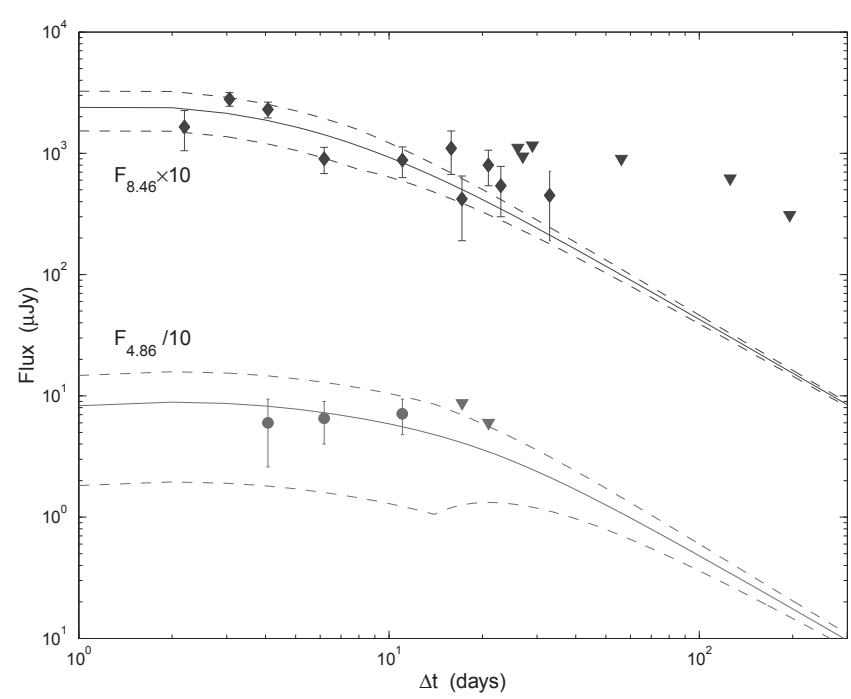

FIG. 5.-Results of the fit to the radio measurements of the afterglow of GRB 000911. The solid line indicates our fit afterglow model light curve, while the dashed lines indicate the scintillation envelope. [See the electronic edition of the Journal for a color version of this figure.]

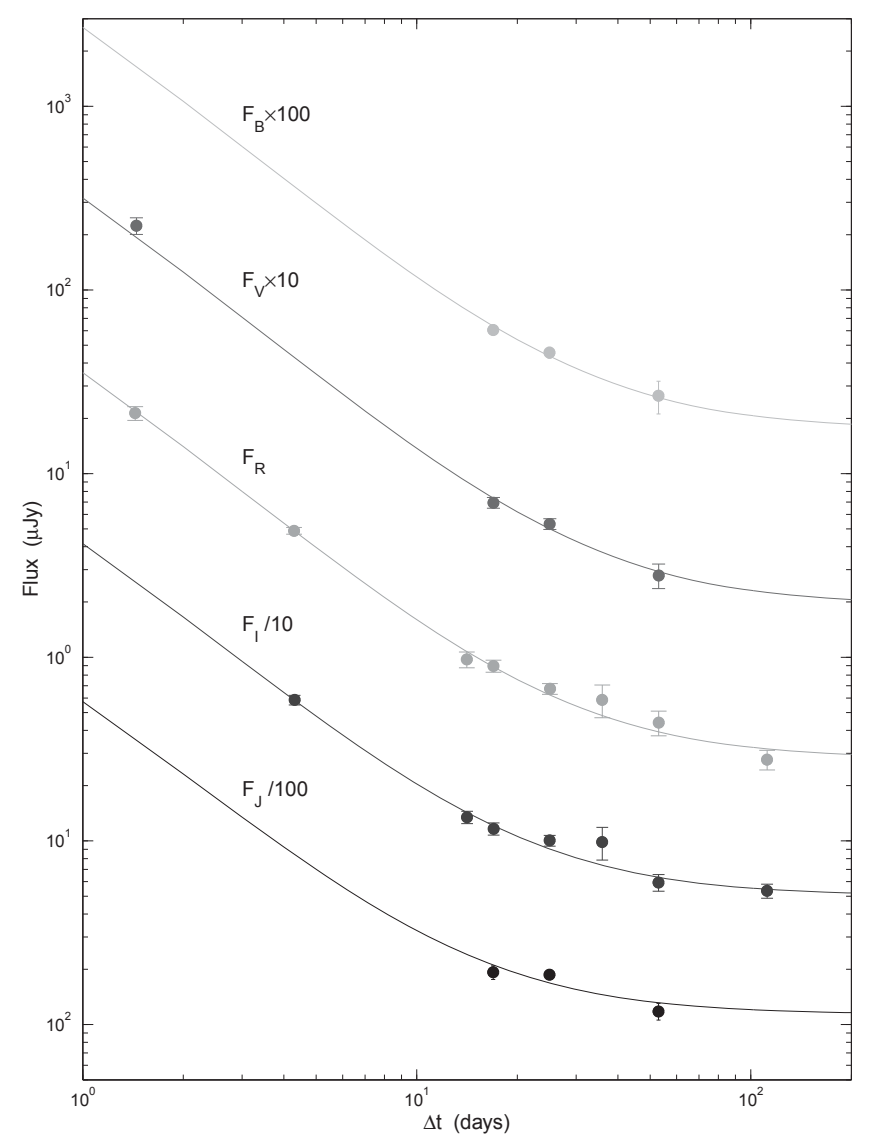

FIG. 6.-Results of the fit to our optical measurements of the afterglow of GRB 000911 and those from Lazzati et al. (2001). The data displayed here have been corrected for Galactic extinction. [See the electronic edition of the Journal for a color version of this figure.]

\section{THE AFTERGLOW MODEL}

We modeled the radio and optical light curves using broadband modeling programs that have been used for other GRBs (see, e.g., Berger et al. 2000; Panaitescu \& Kumar 2000).

The basic afterglow model assumes a relativistic spherical expansion into a constant-density (hereafter ISM; Sari, Piran, \& Narayan 1998) or wind-shaped (hereafter WIND; Chevalier \& Li 2000) medium. In this model, electrons are accelerated at the shock front into a power-law energy distribution, $N(E) \propto E^{-p}$ with $p \sim 2.4$, and emit synchrotron radiation with a spectrum described by three break frequencies and an overall flux normalization $F_{m}$ at the synchrotron peak frequency $\nu_{m}$. The temporal evolution of the spectrum depends on the density $n$ and density structure (ISM or WIND) of the ambient environment. In addition to $p$, the other microphysics parameters are the electron equipartition factor $\epsilon_{e}$ and magnetic field equipartition factor $\epsilon_{B}$.

The ISM model fails to simultaneously fit both the optical and radio data since in this model, $F_{m}$ remains constant with time. As a result, this model predicts a lower flux density level than what is observed in the radio regime. This excess emission is too strong and consistently high to be explained by interstellar scintillation. Explanation in terms of a reverse-shock contribution in the radio at early times (e.g., Frail et al. 2000) also proved to yield unsatisfactory results, with a $\chi^{2} /$ dof $=40 / 31$.

For the WIND model, we again fail to find a satisfactory solution, with our best $\chi^{2}=52$ for 32 dof (we were unable to constrain the cooling frequency in a meaningful way, and so it was fixed). In this case, we encounter a similar problem in which the model predicts a lower flux level than is observed in the radio at early times.

We then considered the ISM model but allowed for a conical geometry (jets) for the explosion (Sari, Piran, \& Halpern 1999). In this model (hereafter ISM + jet), the jet edge becomes visible to observers at epoch $t_{j}$ when the bulk Lorentz factor $\Gamma$ falls below $\theta_{\text {jet }}^{-1}$, the inverse of the jet opening angle. Prior to this jet-break time, the evolution of the synchrotron spectrum is equivalent to the spherical evolution scenarios. For $t \gtrsim t_{j}$, the light curves are expected to be $F_{\nu} \propto t^{-1 / 3}$ or $F_{\nu} \propto t^{-p}$ for $\nu<\nu_{m}$ and $\nu>\nu_{m}$, respectively.

The best-fit parameters for the jet model are $t_{j}=0.6 \mathrm{~d}$, $\nu_{a}=20.5 \mathrm{GHz}, \nu_{m}=460 \mathrm{GHz}, \nu_{c}=8 \times 10^{3} \mathrm{GHz}, f_{m}=4.4$ mJy, and $p=1.49$; here the epoch for the frequencies is $t_{j}$. Thus, the ISM + jet model requires a very "flat $p$ " $(p<2)$. Such flat $p$-values have been inferred in the afterglows of several other GRBs, e.g., GRB 000301C and GRB 010222 (see Panaitescu \& Kumar 2000).

Afterglows with $p<2$ must have a high frequency cutoff so that the total energy in the afterglow does not diverge. Bhattacharya (2001) has shown that the temporal evolution of the spectrum of an afterglow with $p<2$ is identical to that for $p>2$ provided the maximum Lorentz factor of the electron distribution scales linearly with the Lorentz factor of the shock. This requirement has yet to be demonstrated, but appears to be a reasonable assumption. Accepting this ad hoc requirement, we can convert the above inferred parameters to yield the following physical parameters: $p=1.5, E_{52}=1.1, \epsilon_{B}=2.3, \epsilon_{e}=0.016$, and $n=0.07 \mathrm{~cm}^{-3}$. We warn the reader that given the paucity of the afterglow data, each of these values except for $p$ are subject to large errors in the fit break frequencies and should not be taken as 
definitive (the error in $p$ is set by the error in the optical decay slope, which is small); we estimate an error of about a factor of 3-5. The unphysical value of $\epsilon_{B}$ (more than 1 ) is likely the result of these large errors. The overall $\chi^{2} /$ dof $=29.7 / 31$ is quite good, and the results of the fits can be found in Figures 5 and 6 .

\section{HOST GALAXY}

Spectra of the host galaxy were obtained with Keck II on 2001 January 1 UT, in excellent conditions, using the LowResolution Imaging Spectrometer (LRIS; Oke et al. 1995). We used a 1 !.0 wide long slit, at a position angle of $155^{\circ} .1$, close to the parallactic, and a 300 line $\mathrm{mm}^{-1}$ grating, giving an effective instrumental resolution FWHM $\approx 12 \AA$ and an approximate wavelength coverage of 3950-8900 A. We obtained four exposures of $1800 \mathrm{~s}$ each, with small dithers on the slit. Exposures of internal flat-field and arc lamps were obtained for calibrations. Small wavelength shifts due to the instrument flexure were removed by fitting the wavelengths of strong, unblended night-sky lines. The net resulting wavelength scatter was $\sim 0.2 \mathrm{~A}$. Since no adequate flux calibration was obtained for this night, we used the response curves determined from exposures of standard stars $\mathrm{BD}+17^{\circ} 4708$ and $\mathrm{BD}+26^{\circ} 2606$ from Oke \& Gunn (1983), obtained two nights before with the same instrument setup. We removed the residual flux zero-point uncertainty (due to the differential slit losses and seeing variations) by matching the spectroscopic $B V R$ magnitudes to those determined photometrically; we estimate the net flux zero-point uncertainty to be $\sim 20 \%$.

The final combined spectrum of the host galaxy is shown in Figure 7. A strong emission line is detected at $\lambda_{\text {obs }}=7673.1 \pm 0.3 \AA$. This line is also visible in the twodimensional spectrum. Given the presence of a well-detected continuum blueward of the line and the absence of other
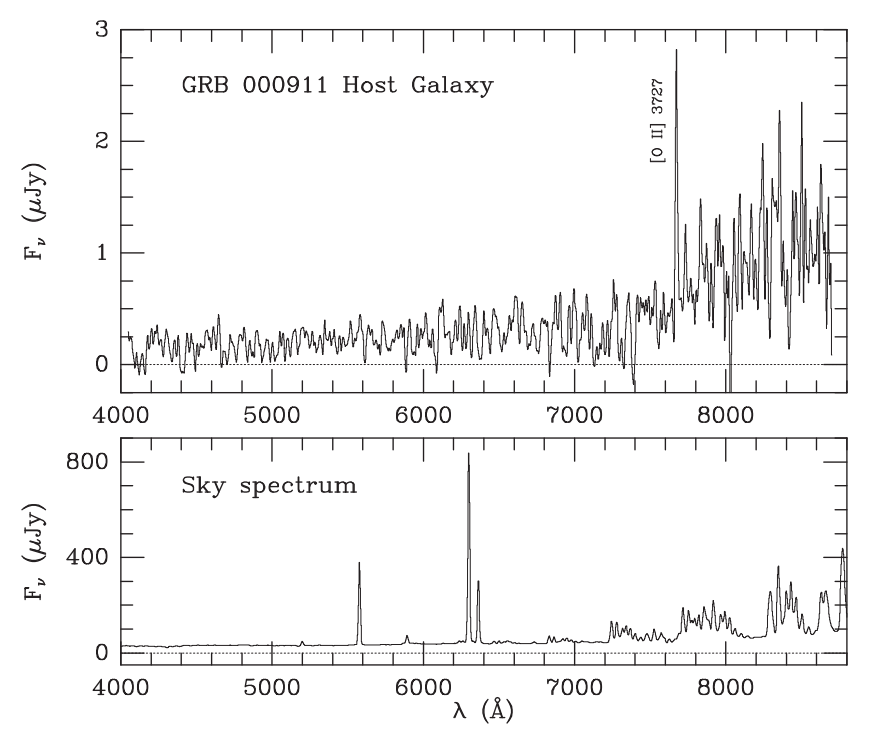

FIG. 7.-Keck spectrum of the host galaxy of GRB 000911 (top), and the corresponding night-sky spectrum (bottom). The spectra have been smoothed with a Gaussian with $\sigma=5 \AA$, corresponding to the instrumental resolution. The [O II] $\lambda 3727$ emission line is labeled; all other apparent spikes are due to imperfect subtraction of the strong night-sky lines. The observed continuum drop near 7500 A corresponds to the Balmer break at the redshift of the object, $z=1.0585$. strong lines, we interpret this line as the commonly detected [O II] $\lambda 3727$ doublet (Djorgovski et al. 2002) at a redshift $z=1.0585 \pm 0.0001$. Note that the instrumental resolution was not high enough to resolve this line as a doublet. There is also a hint of the possible $\mathrm{H} \delta$ line at $\lambda_{\text {obs }} \approx 8442 \AA$, but the difficulty inherent in subtracting the strong night-sky lines in this part of the spectrum precludes a definitive measurement of this or other high-order Balmer lines. However, there is a clear continuum decrement corresponding to the Balmer limit at this redshift, and thus we consider our redshift interpretation to be secure.

In what follows, we assume for the foreground Galactic reddening $E_{B-V}=0.107 \mathrm{mag}$ (Schlegel et al. 1998) and a standard Galactic extinction curve (Cardelli et al. 1989). The fluxes quoted are uncertain by about $20 \%$, with contributions from the statistical noise and the systematic flux zero-point uncertainty of about $10 \%$ each.

The observed [O $\mathrm{II}] \lambda 3727$ line flux ${ }^{14}$ is $(1.9 \pm 0.2) \times 10^{-17}$ ergs $\mathrm{cm}^{-2} \mathrm{~s}^{-1}$. Correcting for the Galactic extinction, we obtain $f_{3727}=(2.3 \pm 0.3) \times 10^{-17}$ ergs $\mathrm{cm}^{-2} \mathrm{~s}^{-1}$. The observed equivalent width is $W_{\lambda}=63 \pm 3 \AA$, i.e., $30.5 \pm 1.5$ $\AA$ in the rest frame. This is typical for field galaxies at comparable redshifts, which have rest-frame equivalent widths spanning 0-50 А (Hogg et al. 1998). The observed continuum flux near $\lambda \approx 5764 \AA$, corresponding to the rest-frame wavelength of $2800 \AA$, is $\approx 0.2 \mu \mathrm{Jy}$. Correcting for the Galactic extinction, we obtain $f_{2800} \approx 0.26 \mu \mathrm{Jy}$. Finally, from the observed $I$-band magnitude of the host of $24.3 \mathrm{mag}$, we estimate the observed flux corresponding to the rest-frame $B$ band to be $\approx 0.45 \mu \mathrm{Jy}$. Correcting for the extinction, we obtain $f_{B} \approx 0.53 \mu \mathrm{Jy}$.

We assume a flat cosmology with $H_{0}=65 \mathrm{~km} \mathrm{~s}^{-1} \mathrm{Mpc}^{-1}$, $\Omega_{M}=0.3$, and $\Omega_{\Lambda}=0.7$. For $z=1.0585$, the luminosity distance is $2.355 \times 10^{28} \mathrm{~cm}$, and $1^{\prime \prime}$ corresponds to 8.7 proper kpc in projection. From the $[\mathrm{O}$ II $] \lambda 3727$ line flux, we calculate the line luminosity $L_{3727}=1.6 \times 10^{41} \mathrm{ergs} \mathrm{s}^{-1}$. Using the star formation rate estimator from Kennicutt (1998), we derive the star formation rate $\mathrm{SFR}_{3727} \approx 2.2 M_{\odot} \odot$ $\mathrm{yr}^{-1}$. From the UV continuum power at $\lambda_{\text {rest }}=2800 \AA$, using the estimator from Madau, Pozzetti, \& Dickinson (1998), we obtain $\mathrm{SFR}_{2800} \approx 1.1 M_{\odot} \mathrm{yr}^{-1}$. The relatively small difference between these two estimates may be due in part to a combination of our measurement errors (which are about $20 \%$ for each value), the intrinsic scatter of these estimators (which is at least 30\%), and possibly some reddening in the host galaxy (the flux at $\lambda_{\text {rest }}=2800 \AA$ would be extincted more than the flux at $\lambda_{\text {rest }}=3727 \AA$ ). Our optically derived SFR is a lower limit since the optical/UV tracers are insensitive to star formation arising in dusty regions.

From the observed continuum flux corresponding to the rest-frame $B$ band, we derive $M_{B} \approx-18.95 \mathrm{mag}$, i.e., the luminosity (then) corresponding to $\sim 0.3 L_{*}$ (today). While the interpretation of this measurement hinges on the assumed evolution of the object (i.e., it may be a progenitor of a present-day spiral or a dwarf galaxy), we note that it is comparable to the observed luminosities of other GRB hosts (Hogg \& Fruchter 1999).

We measure an offset from the afterglow to the host of 0 ". $08 \pm 0$ " 10 , well within the marginally resolved host

14 The fluxes quoted are uncertain by about $20 \%$, with contributions from the statistical noise and the systematic flux zero-point uncertainty contributing about $10 \%$ each. 
(FWHM $\sim 0$ ".72) and consistent with the offsets for other GRBs found by Bloom, Kulkarni, \& Djorgovski (2002). Thus, even in this respect, we find that GRB 000911 appears to be no different from other long/soft GRBs.

\section{CONCLUSIONS}

With a duration of $500 \mathrm{~s}$, GRB 000911 is the longest duration burst to be well localized to date. Here we have presented the high-energy profiles and the evolution of the spectrum. Thanks to a combination of prompt radio and optical observations, we were able to discover and study the afterglow. After the afterglow had faded, we observed the host galaxy and obtained its redshift, $z=1.0585$.

We obtained a modest amount of optical and radio data of the afterglow. The best-fit model requires a flat electron energy index $p \sim 1.5$ and a break in the afterglow at about $\sim 0.6$ days after the burst. Following the usual interpretation, we attribute this break to a jet. At a redshift of 1.0585, the isotropic gamma-ray energy release (using the Konus fluence) is $7.8 \times 10^{53} \mathrm{ergs}$. Using the particular formulation given in Frail et al. (2001), the jet-break time of 0.6 days translates to a jet opening angle $\theta_{j}=19.9$. The gamma-ray energy corrected for the conical geometry is $E_{\gamma} \sim 3 \times 10^{50} n_{0.1}^{1 / 4}$ ergs (Sari et al. 1999), where $n_{0.1}$ is the ambient density in units of $0.1 \mathrm{~cm}^{-3}$. This value is consistent with the median $\left\langle E_{\gamma}\right\rangle$ found by Frail et al. (2001) of $5 \times 10^{50}$ ergs with a $1 \sigma$ spread of a factor of 2 . Since the jet break must have occurred prior to our first observation, a more conservative value of $t_{j}<1.5$ days yields an upper limit on the jet-corrected energy release of $\lesssim 4.2 \times 10^{50} n_{0.1}^{1 / 4} \mathrm{ergs}$, again consistent with the result of Frail et al. (2001).

The properties of this afterglow are quite similar to that of the much shorter duration burst $\left(T_{90} \sim 2 \mathrm{~s}\right)$ GRB 000301C. Panaitescu (2001) finds from broadband modeling of this afterglow that the only way to obtain the rapid decay in the optical bands compared to the radio is to invoke a hard electron distribution. If this is correct, it appears that the duration of the burst has little effect on either the jet opening angle or on the afterglow parameters.

Following the discovery of the afterglow of GRB 000911, Lazzati et al. (2001) organized a program at ESO and Keck to follow up the optical afterglow at late times and found marginal evidence for an underlying supernova (SN). Such late-time red bumps were first noted in GRB 980326 (Bloom et al. 1999) and GRB 970228 (Reichart 1999; Galama et al. 2000) and interpreted as underlying SNs. Other authors have attributed such bumps to dust echoes (Esin \& Blandford 2000). In any case, the presence of such late-time red bumps seem to occur in GRBs with different durations. Finally, the host galaxy of GRB 000911 appears to be quite representative of host galaxies.

We conclude that there is no compelling reason to believe that the duration of GRBs (when restricted to the subclass of long-duration events) either plays a significant role in determining the afterglow properties or perhaps is even particularly related to the properties of the central engine (the jet opening angle).

S. R. K. and S. G. D. thank NSF for support of our ground-based GRB observing program. We are grateful to the staff of the Mount Stromlo and Keck Observatories for their expert help. P. A. P. gratefully acknowledges an Alex Rodgers Travelling Scholarship. J. S. B. gratefully acknowledges the fellowship support from the Fannie and John Hertz Foundation. K. H. is grateful for Ulysses support under JPL contract 958056, and for IPN support under the NEAR Participating Scientist program, NAG 5-9503, and under the LTSA, NAG 5-3500. The Konus/Wind experiment was supported by RFBR grant 99-02-017031 and CRDF grant RPI-2260.

Band, D., et al. 1993, ApJ, 413, 281

Berger, E. J., et al. 2000, ApJ, 545, 56

Bessell, M. S., \& Germany, L. M. 1999, PASP, 111, 1421

Bhattacharya, D. 2001, Bull. Astron. Soc. India, 29, 107

Bloom, J. S., Kulkarni, S. R., \& Djorgovski, S. G. 2002, AJ, 123, 111

REFERENCES

Bloom, J. S., Kulkarni, S. R., \& Djorgovski, S. G. 2002, AJ, 123, 111
Bloom, J. S., et al. 1999, Nature, 401, 453

Cardelli, J. A., Clayton, G. C., \& Mathis, J. S. 1989, ApJ, 345, 245

Chevalier, R. A., \& Li, Z.-Y. 2000, ApJ, 536, 195

Costa, E., et al. 1997, Nature, 387, 783

Djorgovski, S. G., Gal, R. R., Odewahn, S. C., DeCarvalho, R. R., Brunner, R., Longo, G., \& Scaramella, R. 1999, in Wide Field Surveys in Cosmology, ed. S. Colombi et al. (Gif-sur-Yvette: Editions Frontières), 89

Djorgovski, S. G., et al. 2002, in Proc. IX Marcel Grossmann Meeting, ed. V. Gurzadyan, R. Jantzen, \& R. Ruffini (Singapore: World Scientific), in press

Esin, A. A., \& Blandford, R. D. 2000, ApJ, 534, L151

Frail, D. A., et al. 2000, ApJ, 538, L129

.2001, ApJ, 562, L55

Frail, D. A., Kulkarni, S. R., Nicastro, S. R., Feroci, M., \& Taylor, G. B. 1997, Nature, 389, 261

Fryer, C. L., Woosley, S. E., Herant, M., \& Davies, M. B. 1999, ApJ, 520, 650

Galama, T., et al. 2000, ApJ, 536, 185

Henden, A. 2000, GCN Circ. 800 (http://gcn.gsfc.nasa.gov/gcn/gcn3/ 800.gen 3 )

Hogg, D. W., Cohen, J., Blandford, R., \& Pahre, M. 1998, ApJ, 504, 622 Hogg, D. W., \& Fruchter, A. S. 1999, ApJ, 520, 54

Hurley, K., et al. 2001, GCN Circ. 791 (http://gen.gsfc.nasa.gov/gen/ $\mathrm{gcn} 3 / 791 . \mathrm{gcn} 3)$
$.2002, \mathrm{ApJ}, 567,447$

Janka, H.-T., Eberl, T., Ruffert, M., \& Fryer, C. L. 1999, ApJ, 527, L39

Kennicutt, R. C. 1998, ARA\&A, 36, 189

Kouveliotou, C., Meegan, C. A., Fishman, G. J., Bhat, N. P., Briggs, M. S.,

Koshut, T. M., Paciesas, W. S., \& Pendleton, G. N. 1993, ApJ, 413, L101 Lazzati, D., et al. 2001, A\&A, 378,996

MacFayden, A., \& Woosley, S. E. 1999, ApJ, 524, 262

Madau, P., Pozzetti, L., \& Dickinson, M. 1998, ApJ, 498, 106

Nakar, E., \& Piran, T. 2002, MNRAS, 331, 40

Narayan, R., Piran, T., \& Kumar, P. 2001, ApJ, 557, 949

Oke, J. B., \& Gunn, J. E. 1983, ApJ, 266, 713

Oke, J. B., et al. 1995, PASP, 107, 375

Paciesas, W. S., et al. 2000, The Current BATSE Burst Catalog (Greenbelt: Compton Obs. Sci. Support Center)

Panaitescu, A. 2001, ApJ, 556, 1002

Panaitescu, A., \& Kumar, P. 2001, ApJ, 560, L49

Reichart, D. E. 1999, ApJ, 521, L111

Sari, R., Piran, T., \& Halpern, J. 1999, ApJ, 519, L17

Sari, R., Piran, T., \& Narayan, R. 1998, ApJ, 497, L17

Schlegel, D. J., Finkbeiner, D. P., \& Davis, M. 1998, ApJ, 500, 525

van Paradijs, J., et al. 1997, Nature, 386, 686

van Putten, M. H. P. M. 2001, preprint (astro-ph/0109429) 